\title{
Tests Designed to Discover Potential Reading Difficulties at the Six-Year Old Level
}

\author{
KATRINA DE HIRSCH, L.C.S.T. \\ Director, Pediatric Language Disorder Clinic, Pediat ric Section, Vanderbilt Clinic, Columbia Presbyterian \\ Medical Centre, New York City.
}

It is a well-known fact that our schools carry a fairly large percentage of educational and emotional casualties, bright children whose life at school is a burden because they suffer from a more or less severe reading disability. We have only to look at the intake of a pediatric-psychiatric clinic or at a sample of the youngsters referred to our child guidance centers to find a sizable number of intelligent children whose somatic complaints or behavioral disturbances developed only after they had been exposed to the experience of continued failure at school.

Since remedial facilities in our private and public: educational institutions are few and far between, we shall have to find ways to prevent the occurrence of reading failure. In order to do this we need tools which enable us to predict with reasonable certainty which youngsters are liable to run into trouble in the first and second grades. Once we select these children we may find that some of them simply need more time in which to mature, but that others might do very well if given. specific techniques right from the start. Careful selection and consistent planning on our part might easily prevent a great deal of heartache and frustration later on.

How then can we find out at the end of the kindergarten year, in the five-to-six-year-old group - and $I$ stress this time since it is crucial as far as certain maturational processes are concerned - which children are liable to find the going rough?

The remarks offered here are of an entirely tentative nature.

At the Pediatric Language Disorder Clinic, Columbia-Presbyterian Medical Center, we believe that we have evolved some procedures designed to predict future reading performance and we think that in a fair percentage of cases our prediction has been correct. We have not as yet done a statistical evaluation, but having experimented along certain lines, we are now trying to find out where such experimentation will lead us.

We use the well-known intelligence tests as an over-all measurement of the child's basic intellectual endowment. Among them the Bellevue-
Wechsler Scale for Children seems to be the most satisfactory. However, in a large percentage of cases these tests do not predict future success or failure in reading, spelling and writing. The better-known reading readiness tests, which we also use, do not seem to us to cover all the facets of behavior which we think are significant. The Metropolitan Readiness test is probably the best since it stresses comprehension not only of single words, but also of more complex verbal units. It does not, however, test ability to use verbal material and it fails to evaluate a variety of aspects which enter into reading performance. We agree with Gillingham's observation that children with a mental age under $6 \frac{1}{2}$ are not ready for the printed word (5).

Reading readiness is a function of development. We look on development, emotional and neurophysiological, as a progressive increase in complexity of behavioral patterns. Studies on normal development show that psychological functioning and cerebral organization reveal a steady increase in differentiation and integration "through adolescence. As the child grows older he has to cope with increasingly more differentiated and highly integrated organizations. "Among these more complex skills is the ability to use verbal tools. At the age of six children "are supposed to have mastered oral symbols. We expect them to have organized an enormous number of arbitrary phonetic signs into the pattern of language, a formidable achievement which by no means all children have accomplished at this age, as evidenced by the numerous youngsters who still show infantile speech patterns.

Once a child reaches first grade he is expected to cope with a secondary symbolic system, with visual signs which have to be correlated with meaning. In order to read a little word like "hat," a sequence of letters seen, a sequence in space has to be translated into a sequence of sounds heard, a sequence in time. We feel that without a measure of maturation - perceptual, motor,

- The euthor wents to express her gratitude to Dr. Willam s. Langford for his constant and unfalling help and suidence. 
conceptual and behavioral maturation - the child will be unable to cope with this task. The youngster whose neurophysiological organization still is primitive, the one whose language equip. ment is inferior, is the one who will probably run into trouble in the first and second grades. It is this often very intelligent child whom we have to single out before he is exposed to reading failure.

Children between $5 \frac{1}{2}$ and $6 \frac{1}{2}$ usualy make dramatic strides in over-all maturation, strudes so dramatic, in fact, thiot one occasionally feel: one can literally see then blossoming forth. The best time to test them, threfore, is at the end of their year in kindergarte:

Since maturation and development involve the whole child, we observe the yuungster: tolal behavior in order to determine reading readiness.

We usually direct our attention first to those who have trouble with integration of coordination. Movement, like perception, requires patterning. A certain level of motor skills is not only essential for learning to write and print, but it is also indicative of the child's over-all maturity.

The youngsters I refer to do not usually show the severe deviations in large muscular control which we find in cerebral palsied children, but they sometimes have trouble throwing a ball, riding a bicycle, skipping rope. We ask our children to throw darts, to walk on their heels, to hop on one foot. We do occasionally find some who not only have difficulty with the execution of these movements, but who also fail, as in ideomotor apraxia, to get the idea of the act itself. Their over-all motility is often like that of younger children, and it is of interest that Bender and Yarnell (2) have commented on motility disturbances in children suffering from various forms of language disabilities.

Some have not attained the neurological maturation which enables them to execute movements of specific muscle groups. They retain some of the characteristics of the global, total motor response which is typioal for the very young child. For instance, they turn the whole head when asked to flex the tongue.

Lags in integration and patterning of finer muscular control and a degree of dyspraxia, as described by Orton (12), are observed relatively frequently, though occasionally this dyspraxia is confined to graphic activities. Oseretsky's tests(4) provide an extensive survey of muscular skills and have been standardized in terms of normal development. They are thus useful in determining in which areas a child's performance lags. Unfortunately, these tests are difficult to admin ister and for practical purposes we have to rely on our own observations. We give our children identical tasks to perform in order to get a basis for comparison. We watch for jerkiness and arrhythmicity in the smaller muscles of the hand and tongue. The way in which a child handles construction toys will not only reveal his manual dexterity and the fluidity of his movements, but it also provides opportunities for observing many other facets of his behaviour: his span of attention, his frustration threshold, his curiosity and his zest.

Research of the last ten years - especially Werner's work (17) - has revealed the close relationship between perceptual and motor func. tioning. Basic to reading, of course, is the child's ability to cope with perceptual organization.

The very young child normally has difficulty in breaking up the totality of a pattern; his perceptual organization is somewhat diffuse; single parts are poorly differentiated. However, the differentiation of small details and the understanding of the essential relationships between the parts and the whole are a sine qua non in reading.

In the Metropolitan Readiness test we find a few items which require the child to discriminate between small visual details. In fact, much of the reading readiness work done in kindergarten and in the earlier part of the first grade is dedicated to the training of such discrimination, and any of the books used in this readiness work can be used for testing.

The Bender Gestalt test (1), designed for evaluation of visuomotor functioning, is one of the most important in our battery. Visually perceived configurations are offered to children with the request that they be copied. Obviously the infant does not experience perception as the adult does. But the child who is expected to read and write must have visuomotor experiences similar to those of the adult.

Bender says the evolution of visuomotor gestalten is a maturational, not an educative process. It is true that the average 6-year-old does not usually copy all the figures correctly. Developmentally there is a progression in the performance of the copied patterns from a controlled scribble at age 3 to all figures clearly perceived and reproduced at age 11. However, as Silver (13) points out, it is of interest that many of our highly intelligent dyslexic children are unable to cope with this task even at 12 or 13 . They are unable to grasp or retain visual patterns made up of discrete elements. In our children we not only observe difficulty with the handling of the pencil and trouble with manual control, but we also see immature forms (loops, perhaps, instead of dots) which are characteristic for the ages of 4 and 5 . We note verticalization of horizontally oriented figures. We find, in other words, inability to correctly perceive and reproduce given configura- 
tions - functions required in the reading process.

Many of our youngsters have great difficulty spacing these figures on paper. The ability to cope with spatial relationships is of primary importance since in reading and writing the child has to deal with a pattern laid out in space. A developmental lag in this area will thus show up in the youngster's reading performance.

Notions of space originally derive from the child's consciousness of his own body. We use the Goodenough Draw-a-Man test (8) not as an intelligence measurement, nor as a way to evaluate the child's image of self in the emotional sense, though both are of interest. We use it primarily as a relatively reliable indicator of the child's body image, a concept which refers to his awareness of parts of his own body and their relationship to each other. This image is closely related to spatial concepts and is often strikingly immature and primitive in the type of child we are discussing here.

Awareness of left and right, of course, is a significant aspect of the child's notion of space. Since the ability to cope with the specific directional discipline of left to right progression is required for reading in our culture, this aspect deserves further discussion.
As described earlier, we carefully watch the child while we test his finer muscular coordination. Since most of the activities in which he is engaged are untrained and are not influenced by early conditioning, they are useful in determining the degree to which a youngster has established a. functional superiority of one hand over the other. Failure to establish such superiority may be related to familial factors; it may also indicate physiological immaturity and thus tend to show up in reading. We take note of early attempts to switch handedness as well as of family history with regard to laterality. In testing we evaluate strength, precision and speed. We carefully note eye dominance since crossed laterality may adversely influence reading performance.

In the small child, awareness of concepts of right and left progresses slowly. The average child of six can demonstrate right and left on his own body - usually with the help of gestural (motor) responses - but not on anybody else's. That is why Head's finger-to-eye test (9) (originally devised for brain-injured individuals and first used with children by Simon (14) ), which calls for mirror imitation of the examiner's movements, had to be discarded in this form. It seemed to us that the test requires a level of abstraction which the six-year-old has not yet attained. The

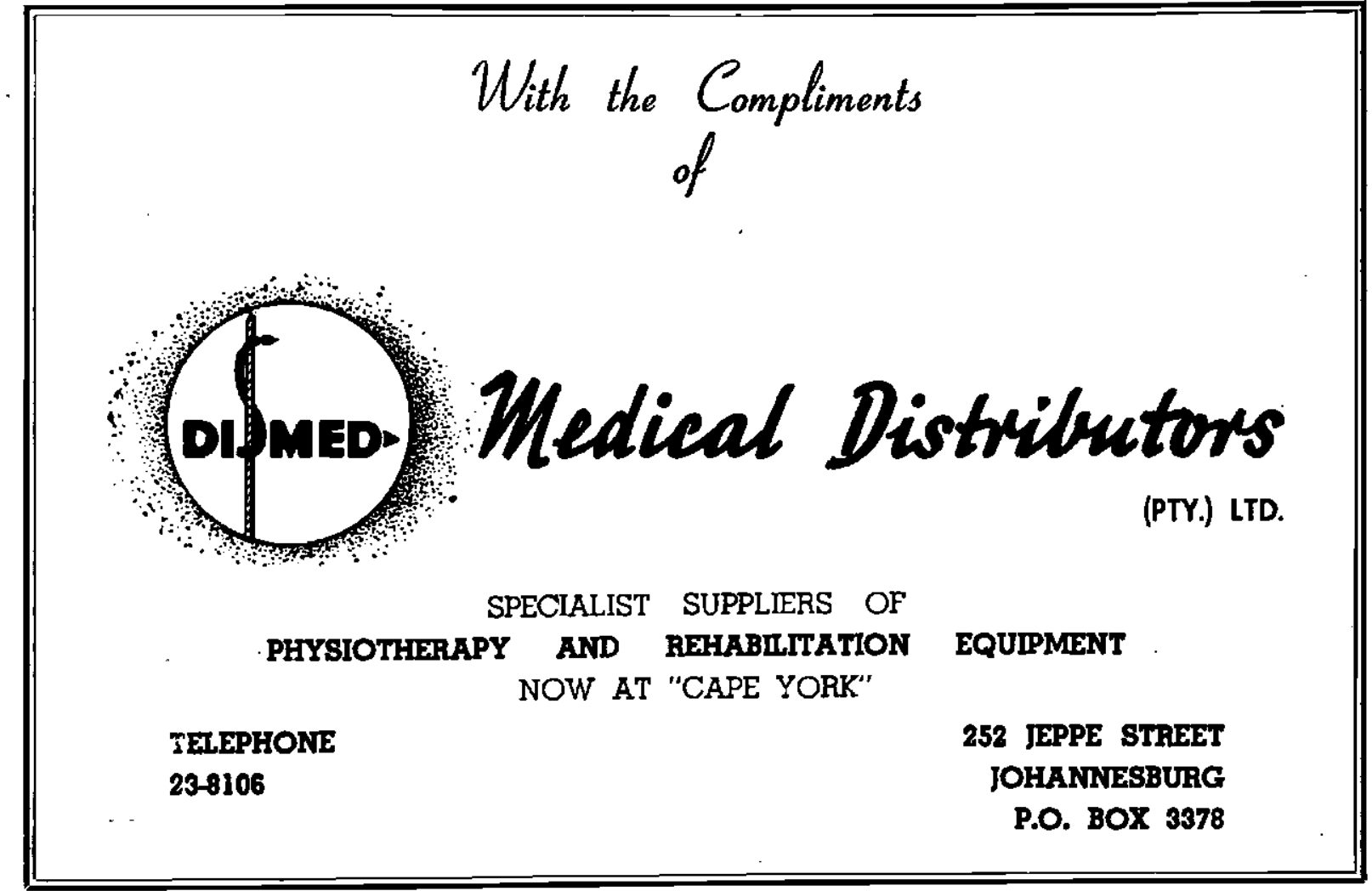


child of six; however, should be able to imitate one's movements when sitting alongside one and watehing one's gestures in a mirror. We have observed that a good many children who fail in this respect later develop reading difficulties.

The Horst's tests (14), which we include in our battery, are useful because they give us a clue as to whether or not the youngster is able to discriminate between identical shapes when they are presented in correct and in reversed form.

Spatial and directional concepts are not the only ones which are pertinent in reading. Language, spoken or printed, is laid out in a time-space pattern. Thus we have to investigate not only spatial but also temporal organization.

It is of interest that a number of workers, like Stambak (15) and Mottier (11), have consistently found rhythmic difficulties in children suffering from reading disabilities, especially in those whose oral language is already somewhat insecure.

We ask our youngsters to imitate tapped-out patterns of varying difficulty and have observed that a goodly percentage of them fail in the repetition of even short and simple sequences. Rhythm is a configuration in time, and funda. mentally our children have trouble with all types of configurations.

We further require that our children repeat a series of nonsense "syllables, and find in the large majority of cases that they have strikingly short auditory memory spans. The correlation of this feature with language disability seems especially high. The child of six should be able to repeat at least four or five syllables, but most of our youngsters manage just three.

There is another area which has so far received little attention and which is closely related to perceptual organization. We know that brain injury, and in fact any lowering of integrative efficiency, brings about an impairment in figurebackground relationships. Weaknesses in figureground relationships have not been systematically explored in children with severe reading disabilities. However, the indications are that these youngsters, though to a lesser degree than do brain.injured ones, have difficulties in this area.

In order to cope with spoken or printed language the child must be able to pick out the figure from the background. For one to interpret a sentence heard (the spoken configuration), the message (like the tapped-out pattern) must stand out clearly. For one to decipher a printed sentence, the configuration must be well defined and sharply delineated against the page. I have seen innumerable youngsters look at printed material as if it represented a meaningless design. Only if the figure does stand out will the sentence or the phrase have structure, or, in other words, meaning. Some of our children have trouble separating figure from background. They do not discover, for instance, the significant design in one of the puzzles when asked to find the lion in the jungle. If one gives them raised geometric figures to reproduce graphically from touch, one often finds that they are drawn to the background, to the roughness of the cloth, for instance. The Marble Board test, which was originally designed to evaluate figure background relationships in brain-injured children, is difficult to administer. At the clinic we therefore use an adaptation of the Figure Background cards presented in Strauss and Lehtinen's book on brain-injured child (16).

It has been established by Goldstein's work (6) that a certain measure of abstract functioning is a requisite of language performance. The question then arises, What does abstract functioning mean at this early age?

We know that the formation of abstract rela. tionships is a developmental process which starts with perceptual and configurational relationships and develops in the direction of - conceptual classification. This applies to both nonverbal and verbal behaviour.

The small child who tries to use the toy toilet

\section{HAVE YOU READ THESE \\ IMPOATANT BOOKS?}

Westerman \& Ward:

Practical Phonetics for Students of South

African Languages.

Van Riper:

Speech Correction, Principles \& Methods.

Anderson:

Improving the Child's Speech.

McCullogh:

Speech Improvement Work and Practice Book

\section{University Bookshop}

Central News Agency Ltd.

Phineas Court

34 Bertha Street

Bracmiontein

Phone 44-7523 : 44-5185 
for his own use while playing with it has not yet understood that a toy only represents the real thing. This experience, a nonverbal one, comes later. It is a tremendous step forward when the child first "pretends" to be $a$ nurse or a pilot. Some children are relatively concrete at the age of six. Practically all brain-injured children are. But we find the group which has difficulties with various aspects of language function to be similarly concrete if to a lesser degree - and this concreteness is by no means restricted to the verbal area.

In order to test abstract behaviour on a nonverbal level, we give our youngsters block designs to copy, and watch whether they are able to analyze wholes into parts and as the next step to synthesize these parts into wholes. Guided by the Goldstein-Scherer tests (7), we give them a variety of objects to sort out, and observe whether they are able to isolate eating utensils, "things to eat with", so as to find out whether they have some form of categorical behaviour, at least on a perceptual level.

The testing discussed up to this point has been confined to non-verbal tasks; we go from these to verbal ones. The ability to handle verbal tools is basic to reading. In order to cope with visual symbols the child must have mastered auditory ones, oral language. Careful testing in this area often reveals significant gaps which are frequently overlooked. Authors like Orton (12), McCarthy (10), and Borell-Maisonny (3) have long stressed the close relationships between reading efficiency and oral language skills.

First of all we have to make sure that the youngster fully understands spoken material. Units like "in front of," "inside," "beneath," which are fairly abstract concepts, are by no means always as securely established as we are inclined to think. We place the child in front of the dollhouse' and suggest he put the baby next to the bathtub and so on. Carrying out complex directions is not always easy for our children; many are unable to interpret a somewhat involved story; as a matter of fact, a few are not ready to listen at $i$ all. Some do not catch on when presented with an absurdity couched in verbal terms, although they are easily able to see the point if the absurd is presented in pictorial form.

Comprehension of language is one thing; use of language, another. We carefully check on articulatory patterns; we note length of units and listen for difficulties in word finding. There are children who have a relatively good use of the idiom on an auditory perceptual basis; they sound as though they have a large vocabulary, but some of them fail when asked to give a word on being presented with a picture.

We are interested in vocal patterns - an unusual degree of monotony may reflect a diff. culty with structuralization, perhaps a figurebackground problem.

The child's ability to form correct grammatical constructions is of importance. Grammar is an expression of structure, and the child who leaves out small connectives in the sentence may have difficulties with the temporal, spatial and causal relationships expressed by these words.

We further want to know whether the youngster is able to tell a simple story and to bring out its salient features. Some children's organization of verbal material is so poor that they never get their point acrossi they get so involved in the intricacies of the "Three Little Pigs" that they ramble on indefinitely.

In the beginning the child's spoken language is on a very concrete level. A three-year-old who says "brush" does not refer to the category "brush," to the object whose essential qualities are unchanging from situation to situation. "Brush" to him might mean one time, "Brush my hair"; another time, "The brush is on the table." He does not use the word in a categorical sense.

In testing abstract functioning on a verbal level we look for the youngster's ability to classify and categorize. We ask him to name all diningroom furniture. If he includes the wallpaper or the silver dishes on the sideboard he shows thereby that he has not yet understood the category "furniture." Werner (18) cites the example of the boy who includes bread and a pipe with the bench, the saw and the hammer when asked to list his tools, explaining, "When you have finished working at the bench you want to eat and smoke a pipe." In other words, he grouped objects according to a concrete situation and not according to the more abstract category "tools." We use the Columbia Mental Maturity Scale to test categorical behaviour.. The intelligent boy who, when shown a picture of a hen, a stove, a pot and an egg, says that the egg and the pot belong together, "because you cook an egg in the pot," indicates that he is unable to free himself from the concrete and is not yet ready for classifications.

We test the child's ability to give definitions. At the age of six children define objects in terms of function. A six-year-old who says, "You hold it up here," when asked what a violin is, behaves in a very concrete way, which, if persisting, is a poor prognosis in terms of academic functioning.

If one asks a number of six-year-old children what a policeman is, one is apt to receive a variety of answers from: "He wears a blue suit," or "He stands at the corner" (which are concrete 
responses), to "He directs traffic." In the last response the child tries to cope with function, while in the first one he limits himself to description It is usually the child with a language difficulty who is more concrete than others are.

Most children who later develop reading disabilities seem to have trouble with patterning the units of wonds and sentences in spoken speech. Orton (12) has shown how frequently these youngsters tend to reverse both oral and printed symbols. The same boy who says "Crice Ripsies" for "Rice Crispies" is the one who later on reads "was" for "saw" and "now" for "won."

Reversal and confusion in the order of sequences - which are closely related to temporal organization in the sense in which it has been discussed - are usually not confined to syllables and words. The whole sentence is often jumbled, showing again that it is in the area of organization and structuralization of both short and long units that children with language deficits have outstanding difficulties.

Most of our tests are designed to measure the child's ability to pattern, structuralize, and adequately respond to the endless stream of stimuli to which he is exposed at every moment. However, organization of perceptual and motor patterns is not the only area which presents difficulties for our children. Many of them have trouble with integration of behavior. The result is hyperkinesis and lack of control. Most children suffering from developmental language lags are enormously hyperactive. Their trouble with inhibition and channeling of impulses seems to be but another aspect of their inability to organize stimuli (arising from inside as well as from outside) into behavioral configurations. Hence they find it difficult to sit still several hours a day. Such children (they may or may not be emotionally disturbed) are bound to have trouble concentrating. Since they are unable to exclude a variety of stimuli, they are incapable of focusing their attention on a specific gestalt, or an assigned task.

Children in kindergarten are usually given a good many motor outlets, but once they get into first grade they find it difficult to cope with a more structured framework, since their frustration tolerance is low and their need for large muscular activity considerable.

Among the children whom we have tested during the last few years we have found a fairly steady, though small, number of youngsters whose perceptual deviations, trouble with figure-background relationships, outstandingly poor motor perform ance and limitation in abstract behavior seem far more severe than is usual for the child who suffers from a developmental language disability. Careful investigation of these cases has sometimes revealed a positive history, for instance, anoxia at birth. These youngsters do not necessarily show their usual positive signs on the classical neurological examination. However, more refined testing procedures show that they have difficulties at various levels of integration. Watching these children copy the Bender gestalt figures, one often finds a marked tendency to disinhibition, accompanied by compensatory rigidity. We find perseveration in various areas and a tendency to go to pieces when the number of stimuli becomes too great. Many of these relatively subtle signs go undiscovered until the time when these children are confronted with a task which is as complex as is the mastering of oral and printed symbols.

I do not want to give the impression that our testing takes a great deal of time. After some experimenting we have brought the time down to between 40 and 45 minutes. The tests are usually administered during one session, or, preferably, two. Careful observation of the child as he functions in kindergarten, moreover, will eliminate many of the more formal procedures.

In conclusion I should like to sum up a few points: Maturation is largely a process of integra. tion and differentiation. The child of six and older whose perceptual, motor, visuomotor and conceptual performance is still relatively primitive, the child who has trouble with structuralization of behavioral patterns, is the one who is liable to run into difficulties when he is exposed to reading, which requires the smooth interplay of many facets of behaviour.

Some of these children need more time in which to mature. Postponing formal training and discipline for 6 to 12 months may prevent future regrading with its attending experience of failure and humiliation which might easily spoil the youngster's entire learning pattern.

But our testing should do more than simply pick out the children who are not as yet ready for first grade. It should assist us in determining what type of help would be suitable for those youngsters who we feel are able to make the grade if given specific assistance and support.

The hyperactive child, for instance, needs a teacher who has some tolerance for the child's specific difficulty. He needs, if possible, a setting which allows him a good many motor outlets while at the same time providing a somewhat Istructured environment which protects him from an excess of environmental stimuli. Such a youngster would fare better if seated in the front of the room where he sees only the teacher and not his classmates. 
The child with an oral language disability, on the other hand, whose lags are confined to specific areas, might do all right if he were referred to a speech therapist and helped to establish more speech patterns and a more extensive vocabulary. Another youngster might need assistance with straightening out his confusion in cerebral dominance, and help in establishing left-to-right progression.

Most of these children, as Orton (12) has pointed out, usually do better with a phonetic approach to reading than with the whole-word attack. There are good reasons for this fact and they lie precisely in the direction we have discussed. The child who has trouble with the organization of visual patterns is naturally bewildered and confused if he is confronted with what to him seem to be diffuse and undifferentiated configurations. He will benefit immensely if words are broken up into small phonetic units. This breaking-up process actually represents a transposal of spatial sequences into temporal ones. In this manner many youngsters are able to cope with single sounds, short auditory configurations which they slowly learn to fuse into larger entities. This procedure facilitates the structuralization of - for them - undifferentiated wholes and thus gives them a larger measure of security.

There are, of course, exceptions. There are children who fail to respond to the phonetic method. These particular children fall into three categories: The hyperactive child does not always possess the span of attention required for the laborious sounding out of words. The process is too slow for him and he tends to get discouraged and frustrated. Into the second category fall the youngsters who have trouble with abstract behaviour. The process of analyzing a word into its parts and then synthesizing the parts requires a certain level of abstraction. Moreover, the very concept that a letter seen represents a speech sound heard is difficult for the brain-injured child (who is usually hyperactive as well) to grasp. In the third category falls the youngster who shows obsessive tendencies. He will stick compulsively to single sounds; he will be too anxious to blend them successfully into words or to integrate them into meaningful sentences.

Thus the tests for prediction of future reading disabilities are not only designed to discover the child who is liable to run into trouble with reading, but are also meant to indicate the areas in which a child's performance lags. The tests should actually do more: they should provide a lead as to what specific techniques could be used to advantage in future training.

Precious time is thus saved, and some children, at least, are spared the humiliating experience of failure in reading, writing and spelling (which are all important in the earlier grades), a failure which will often carry over into other learning experiences.

Not all children suffering from potential reading difficulties are primarily emotionally disturbed. However, their basic developmental lag in physiologioal-psychological functioning makes. them especially susceptible to adverse educational experiences and as a result they often develop secondary emotional difficulties very early.

We hope to discover some of these youngsters before they become educational and emotional casualties.

\section{REFERENCES}

1. Bender, L. A. A Visual Motor Gestalt Test and Its Clinical Use. American Orthopsychlatric Assoc, New York, 1938.

2. Bender, L., and H. Yarnell. An Observation Nursery. Am. J. Psychiatry, 97:1158, 1941.

3. Borell-Maisonny, S. Les Troubles da language dans les dyslexies et les dysortographios. Enfance, 5:400, 1951.

4. Doll, E. The Oseretsky Tests of Motor Proficiency. Educational Publishers, Minneapolis, 1940.

5. Gillingham, A. Avoiding Failure in Reading and Spelling. Independent School Bull., Nov. 1949.

6. Goldstein, $K$. Language and Language Disturbances. Grune \& Stratton, New York, 1948.

7. Goldstein, $\mathbf{K}$, and $\mathbf{M}$. Scherer. Abstract and Concrete Behaviour. Psychol. Monogr., 53:1, 1941.

8. Goodenough, F. Measurement of Intelligence by Drawing. World Book, Yonkers-on-Hudson, New York, 1926.

9. Head, H. Aphasia and Kindred Disorders of Speech, Vol. I. Cambridge Univ. Press, London, 1926. McCarthy, D. Chap. 9 in Manual of Chlld Psychology (Leonard Carmichael, §d.). (2nd ed.) Wiley, New York, 1954.

11. Mottier, G. Uber Untersuchung der Spracho lesegestorter Kinder. Folio Phonlatrica, 3: No. 3, 1951.

12. Orton, S. T. Reading, Writing and Speech Problems in Children. Norton, New York, 1937.

13. Silver, A. Diagnostic Value of Three Drawing Tests for Children. J. Pediat, $37: 1,1950$.

14. Simon, J. Contributions a la Psychologie de la Lecture. Enfance, 6:438-447, 1964.

15. Stambak, M. Le Probleme du rhythmo dans lo developpement de lenfant et dans les dyslexies d'evolution. Enfance, 5:480-493, 1951.

16. Strauss, A., and L. Lehtlnen. Psychopathology and Edacation in the Brain-Injured Chlld. Grune \& Stratton, New York, 1947.

17. Werner, H. Towards a General Theory of Perception. Psychol. Rev., 59:5, 1952.

18. Comparative Psychology of Mental Development. Harper, New York, 1940. 